\title{
Rich Spatiotemporal Dynamics of a Vegetation Model with Noise and Periodic Forcing
}

\author{
Xia-Xia Zhao ${ }^{1,2}$ and Jian-Zhong Wang ${ }^{1,2}$ \\ ${ }^{1}$ National Key Laboratory for Electronic Measurement Technology, North University of China, Taiyuan, Shanxi 030051, China \\ ${ }^{2}$ Key Laboratory of Instrumentation Science and Dynamic Measurement, Ministry of Education, North University of China, \\ Taiyuan, Shanxi 030051, China
}

Correspondence should be addressed to Xia-Xia Zhao; zxxzbdx@sina.cn

Received 1 September 2013; Revised 16 December 2013; Accepted 4 January 2014; Published 3 March 2014

Academic Editor: Ryusuke Kon

\begin{abstract}
Copyright ( $\odot 2014$ X.-X. Zhao and J.-Z. Wang. This is an open access article distributed under the Creative Commons Attribution License, which permits unrestricted use, distribution, and reproduction in any medium, provided the original work is properly cited.

The growth of vegetation is undeniably subject to random fluctuations arising from environmental variability and internal effects due to periodic forcing. To address these issues, we investigated a spatial version of a vegetation model including seasonal rainfall, noise, and diffusion. By numerical simulations, we found that noise can induce the pattern transition from stationary pattern to other patterns. More specifically, when noise intensity is small, patch invasion is induced. As noise intensity further increases, chaotic patterns emerge. For the system with noise and seasonal rainfall, it exhibits frequency-locking phenomena. Patterns transition may be a warning signal for the onset of desertification and thus the obtained results may provide some measures to protect vegetation, such as reducing random factors or changing irrigation on vegetation.
\end{abstract}

\section{Introduction}

Understanding the effect of external variability on vegetation systems is a problem of great interest. An obvious and most important source of external variability is seasonality. The consequences of the cyclic variation of seasonality have been well investigated [1-4]. The importance of understanding the effect of the seasonal rainfall on the biological productivity of a region within a specific model is equal to understanding the effect of additional fluctuations such as interannual variations [5]. On the other hand, the environments in models and laboratories are much less complex than ecological environments, and thus vegetation systems can be modeled as open systems in which the interaction with the environment is noisy [6, 7]. Stochasticity can give rise to counterintuitive behaviors such as stochastic resonance, noise-enhanced stability, noisedelayed extinction, and noise-induced pattern formation [810].

In the past years, the influences of noise and periodic forcing have been well studied in ecosystems [11-13]. They found from ecological models that noise can induce the emergence of chaotic patterns. Moreover, noise and external periodic forces may cause instability and enhance the oscillation of the species density. In addition, interactions of noise and external periodic forces can give rise to resonant patterns and frequency-locking phenomena.

Regular patterns of vegetation have been observed in many arid and semiarid regions of the world. The formation of regular vegetation bands on hillsides of semiarid catchments is often attributed to a low scale process of water redistribution by runoff [14-20]. It is well recognized that local feedbacks along with dispersion can induce regular vegetation patterns to develop as a result of Turing-like instability. This phenomenon motivated many recent studies concerning the stability of the soil moisture and plant biomass balance equations [20]. Mathematical analysis showed that, when the vegetation system is unstable, an even slightly heterogeneous initial distribution of the vegetation can induce regular spatial patterns. Now, it is natural to ask whether the noise and seasonal rainfall can induce spatial patterns in vegetation systems.

The main purpose of this paper is to investigate the effects of noise and seasonal rainfall on the vegetation patterns. In 
particular, we want to check whether pattern transition or frequency locking emerges. The rest of our paper is arranged as follows. In Section 2, we give the vegetation model with noise, seasonal rainfall, and spatial diffusion. In Section 3, it is found that there is pattern transition from stationary pattern to patch invasion or chaotic pattern. Furthermore, the interactions of noise and periodic forces can give rise to frequency-locking phenomena. Finally, we present some conclusion and discussion.

\section{Main Model}

Von Hardenberg et al. proposed a partial differential equation model, with equations for the biomass density $n(r, t)$ and the ground water density $w(r, t)$ in both space and time [21]:

$$
\begin{gathered}
\frac{\partial n}{\partial t}=\frac{\gamma w}{1+\sigma w} n-n^{2}-\mu n+\nabla^{2} n \\
\frac{\partial w}{\partial t}=p-(1-\rho n) w-w^{2} n+D \nabla^{2}(w-\beta n) \\
-\nu \frac{\partial(w-\alpha n)}{\partial x}
\end{gathered}
$$

where $\nabla^{2}=\partial^{2} / \partial x^{2}+\partial^{2} / \partial y^{2}$ is the Laplacian operator in two-dimensional space with $r=(x, y) .(\gamma w /(1+\sigma w)) n$ is used to describe plant growth at a rate that grows linearly with $w$ for dry soil; $-\mu n$ presents mortality and herbivory; $-n^{2}$ represents saturation due to limited nutrients; $\nabla^{2} n$ is the spread of plants; $p$ corresponds to precipitation; $-(1-\rho n) w$ is evaporation; $-w^{2} n$ is local uptake of water by plants; $D \nabla^{2}(w-$ $\beta n)$ is the feedback term; $\nu(\partial(w-\alpha n) / \partial x)$ is used to model the drop of runoff in vegetated areas due to increased infiltration.

When combined with noise and seasonal rainfall, the original spatially extended model is written as the following system:

$$
\begin{gathered}
\frac{\partial n}{\partial t}=\frac{\gamma w}{1+\sigma w} n-n^{2}-\mu n+\eta(r, t)+\nabla^{2} n \\
\frac{\partial w}{\partial t}=p\left[A_{0}+A_{1} \sin (\omega t)\right]-(1-\rho n) w-w^{2} n+\eta(r, t) \\
+D \nabla^{2}(w-\beta n)-\nu \frac{\partial(w-\alpha n)}{\partial x} .
\end{gathered}
$$

The seasonal rainfall is assumed to be sinusoidal with amplitude $A_{1}$ and frequency $\omega$, which is considered as an additive version for the reason that toxins produced by different populations have a significant role in shaping the dynamical behavior of ecosystems [22]. $\omega$ is related to a seasonal rainfall variation. There are 12 months in one year and rainfall may be different in different months, so the value of $\omega$ is $\pi / 6$. In that case, the rainfall reaches its maximum value in March and minimum value in September. This phenomenon can be found in Southern China.

In $(2 \mathrm{a})$ and (2b), the stochastic factors are taken into account as the term $\eta(r, t)$, which is obtained in the continuum limit from the master equation arising from microscopic interaction in the space $[23,24]$ where the typical white noise will emerge. Recently, colored noise and white noise have both been used in describing ecological evolution $[25,26]$. White noise is the limiting case of colored noise, so we consider the more general case-colored noise in the present paper. The noise term $\eta(r, t)$ is introduced additively in space and time, which is the Ornstein-Uhlenbech process [13, 27]. The colored noise $\eta(r, t)$, which is temporally correlated and white in space, satisfies

$$
\left\langle\eta\left(r_{1}, t_{1}\right) \eta\left(r_{2}, t_{2}\right)\right\rangle=\frac{\varepsilon}{\tau} \exp \left(-\frac{\left|t_{1}-t_{2}\right|}{\tau}\right) \delta\left(r_{1}-r_{2}\right),
$$

where $\tau$ controls the temporal correlation and $\varepsilon$ measures the noise intensity. Here, \langle\rangle means inner product and $\delta$ presents impulse function.

Before proceeding to the spatially explicit case, the first step is to have a look at the properties of the local dynamics. The local system of systems (1a) and (1b) is as follows:

$$
\begin{gathered}
\frac{d n}{d t}=\frac{\gamma w}{1+\sigma w} n-n^{2}-\mu n=H_{1}(n, w), \\
\frac{d w}{d t}=p-(1-\rho n) w-w^{2} n=H_{2}(n, w) .
\end{gathered}
$$

We assume that the local system has a stable equilibrium $E^{*}=\left(n^{*}, w^{*}\right)$ which can be obtained by solving $H_{1}(n, w)=0$ and $H_{2}(n, w)=0$. The Jacobian matrix corresponding to this equilibrium point is

$$
J=\left(\begin{array}{ll}
a_{11} & a_{12} \\
a_{21} & a_{22}
\end{array}\right)
$$

We make the following substitute: $n=n^{*}+\bar{n}(\vec{r}, t)$ and $w=w^{*}+\bar{w}(\vec{r}, t)$, into systems (1a) and (1b), and assume that $|\bar{n}| \ll n^{*},|\bar{w}| \ll w^{*}$. Then, in the linear approximation, we obtain that

$$
\begin{gathered}
\frac{\partial \bar{n}}{\partial t}=a_{11} \bar{n}+a_{12} \bar{w}+\nabla^{2} \bar{n}, \\
\frac{\partial \bar{w}}{\partial t}=a_{21} \bar{n}+a_{22} \bar{w}+D \nabla^{2} \bar{w} \\
-D \beta \nabla^{2} \bar{n}-\nu \nabla \bar{w}+\alpha \nu \nabla \bar{n} .
\end{gathered}
$$

The initial conditions are assumed as $\left.\bar{n}\right|_{t=0}=f(\vec{r})$ and $\left.\bar{w}\right|_{t=0}=g(\vec{r})$, where the functions $f(\vec{r})$ and $g(\vec{r})$ decay rapidly for $\vec{r} \rightarrow \pm \infty$. Following the standard approach, let us now perform a Laplace transformation of the linearized equations over the two independent variables $\vec{r}$ and $t$. For $\vec{r}$ we use the so-called two-sided version of the transformation. The relations for the forward and backward transforms are $[28,29]$

$$
\begin{gathered}
n_{\lambda q}=\int_{0}^{\infty} e^{-\lambda t} d t \int_{-\infty}^{+\infty} \bar{n}(\vec{r}, t) e^{-q \vec{r}} d \vec{r}, \\
\bar{n}(\vec{r}, t)=-\frac{1}{4 \pi^{2}} \int_{\beta-i \infty}^{\beta+i \infty} e^{\lambda t} d \lambda \int_{-i \infty}^{i \infty} n_{\lambda q} e^{q \vec{r}} d q,
\end{gathered}
$$


where $\lambda$ and $q$ are complex variables. After this transformation, we have that

$$
\begin{aligned}
& \left(\lambda-a_{11}-q^{2}\right) n_{\lambda q}-a_{12} w_{\lambda q}=F(q), \\
& \left(\lambda-a_{22}-D q^{2}+v q\right) w_{\lambda q} \\
& \quad+\left(-a_{21}+D \beta q^{2}-\alpha v q\right) n_{\lambda q}=G(q),
\end{aligned}
$$

where $F(q)$ and $G(q)$ are the transforms of $f(\vec{r})$ and $g(\vec{r})$. The transition between these two cases happens at the critical advection coefficient that can be determined from the set of equations $[28,29]$

$$
L(\lambda, q)=0
$$

By solving the linear equations (9) and (10) we find $n_{\lambda q}$ and then use the backward transformation (8) to obtain the following formal solution:

$$
\begin{aligned}
\bar{n}(\vec{r}, t)= & -\frac{1}{4 \pi^{2}} \int_{\beta-i \infty}^{\beta+i \infty} e^{\lambda t} d \lambda \\
& \times \int_{-i \infty}^{i \infty} \frac{\left(\lambda-a_{22}-D q^{2}+v q\right) F(q)+a_{12} G(q)}{L(\lambda, q)} e^{q \vec{r}} d q .
\end{aligned}
$$

Then we obtain the linear stability of this state which is described by the dispersion relation:

$$
\begin{aligned}
L(\lambda, q)= & \left(\lambda-a_{11}-q^{2}\right)\left(\lambda-a_{22}-D q^{2}+v q\right) \\
& +a_{12}\left(-a_{21}+D \beta q^{2}-\alpha v q\right) .
\end{aligned}
$$

For the sake of convenience, we firstly pay attention to the one dimension case. By setting $q=i \kappa$, we have that

$$
\lambda=\frac{a_{11}+a_{22}-\kappa^{2}-D \kappa^{2}}{2}+\frac{\nu \kappa i}{2} \pm \frac{\sqrt{C_{1}+C_{2} i}}{2}
$$

where $C_{1}=(D-1)^{2} \kappa^{4}+\left(2 a_{11} D+2 a_{22}-v^{2}-2 a_{22} D-2 a_{11}+\right.$ $\left.4 a_{12} D \beta\right) \kappa^{2}+\left(a_{11}-a_{22}\right)^{2}+4 a_{12} a_{21}$ and $C_{2}=-2 a_{11} \nu \kappa+2 \nu \kappa^{3}-$ $2 D \nu \kappa^{3}+2 a_{22} \nu \kappa+4 a_{12} \alpha v \kappa$.

Straightforward manipulation of (14) yields

$$
\begin{gathered}
\operatorname{Re}(\lambda)=\frac{a_{11}+a_{22}-\kappa^{2}-D \kappa^{2}}{2} \pm \frac{1}{2} \sqrt{\frac{1}{2}\left(\sqrt{C_{1}^{2}+C_{2}^{2}}+C_{1}\right)} \\
\operatorname{Im}(\lambda)=\frac{\nu \kappa}{2} \pm \operatorname{sign}\left(C_{2}\right) \frac{1}{2} \sqrt{\frac{1}{2}\left(\sqrt{C_{1}^{2}+C_{2}^{2}}-C_{1}\right)}
\end{gathered}
$$

where $\operatorname{Re}(\lambda)$ and $\operatorname{Im}(\lambda)$ are the real and imaginary parts of $\lambda$, respectively.

The condition for a spatial mode $q$ (in one- or twodimensional space) is to be unstable and thus systems (1a) and (1b) grow into a pattern; that is, $\operatorname{Re}(\lambda)>0$.

\section{Main Results}

In this section, extensive testing was performed through numerical integration to describe systems (2a) and (2b). In simulation, zero-flux boundary conditions are used and the time step is $\Delta t=10^{-5}$ time unit. The space step is $\Delta x=\Delta y=0.1$ length unit and the grid sizes in the evolutional simulations are $M \times N(M=N=500)$. The Fourier transform method is used for the deterministic part in (1a) and (1b). On the discrete square lattices, the stochastic partial differential equations (2a) and (2b) are integrated numerically by applying the Euler method. Several different discrete methods were checked, and the results indicate that the Fourier transform accurately approximates solutions of (2a) and (2b). On the other hand, the Fourier method offers a speed advantage over other numerical methods.

3.1. Pattern Formation of Systems (1a) and (1b) without Noise and Periodic Force. In order to well show the effects of noise and seasonal rainfall, we pay attention to the spatial pattern of systems (1a) and (1b) without noise and periodic force. Parameters' values are used as $\gamma=1.6, \sigma=1.6, \mu=0.5$, $\rho=1.5, D=10, \alpha=3$, and $\beta=3$, which can ensure that $\operatorname{Re}(\lambda)>0$, for the simulation.

In Figure 1, we show the spatial pattern of the vegetation in two-dimensional space. The initial density distribution corresponds to random perturbations around the trivial stationary state. One can see that, for the cases $p=0.45$ and $\nu=0$, systems (1a) and (1b) can show spotted pattern (see Figure 1(a)). And for the cases $p=0.13$ and $v=50$, the stripelike and spotted patterns coexist in the space (see Figure 1(b)). The pattern structures are consistent with the previous work [30].

For the systems (1a) and (1b), one can see that the random initial distribution leads to the formation of regular patterns, which means that the distribution of vegetation is self-organized.

3.2. Emergence of Pattern Transition of Systems (1a) and (1b) with Noise. In recent years, noise-sustained and noiseinduced spatial pattern formations have been discussed in ecological systems [31, 32]. Now, we firstly investigate the spatial pattern of the systems (1a) and (1b) with $v=0$ when the additive noise is turned on. It can be seen from Figure 1(a) that typical stationary Turing pattern is shown when there is only diffusion. However, combined with additive noise, Turing pattern disappears and crescent moon-like pattern emerges (cf. Figure 2(a)). When the noise intensity and temporal correlation further increase, patch invasion will emerge which can be seen from Figure 2(b).

For the case $\nu \neq 0$, we found that there is chaotic pattern for some appropriate values of noise intensity and temporal correlation shown in Figure 3. Chaos may lead to the extinction of or disorder in the vegetation density [19], which implies that noise may induce the onset of desertification.

In order to see the effects of noise intensity and temporal correlation on pattern dynamics, we give regions of pattern structures with respect to the two parameters in 


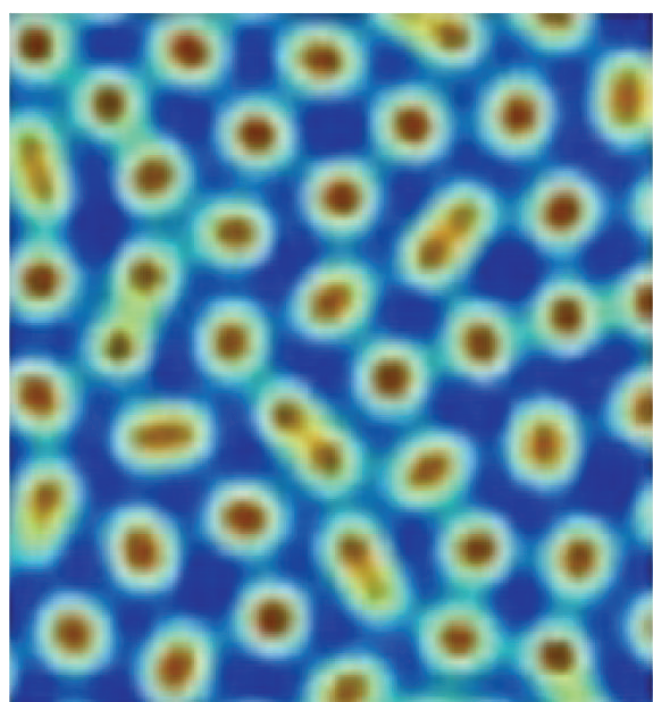

(a)

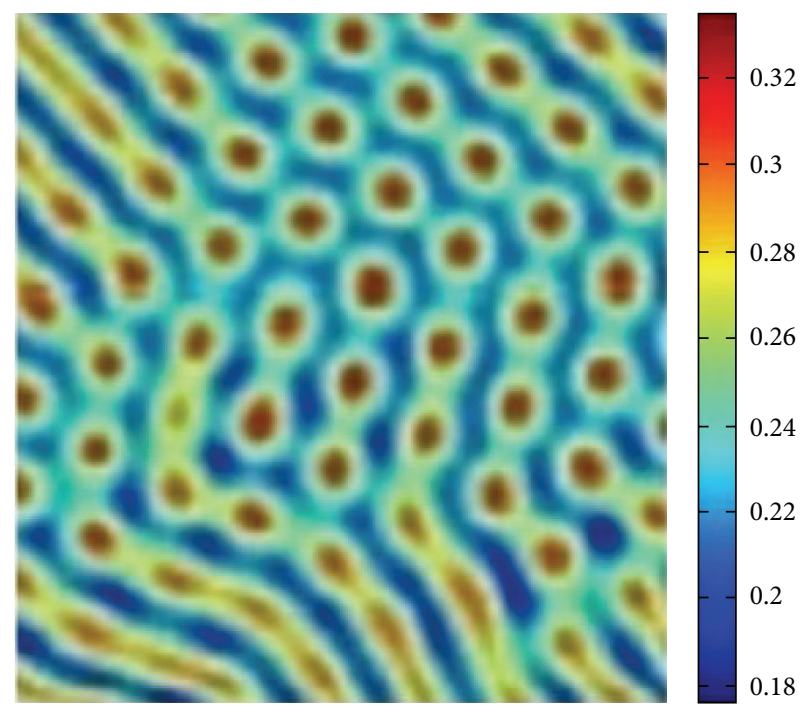

(b)

Figure 1: Snapshots of contour pictures of the vegetation at $t=1000$ with $\gamma=1.6, \sigma=1.6, \mu=0.5, \rho=1.5, D=10, \alpha=3$, and $\beta=3$. (a) $p=0.45$ and $\nu=0$; (b) $p=0.13$ and $\nu=50$.

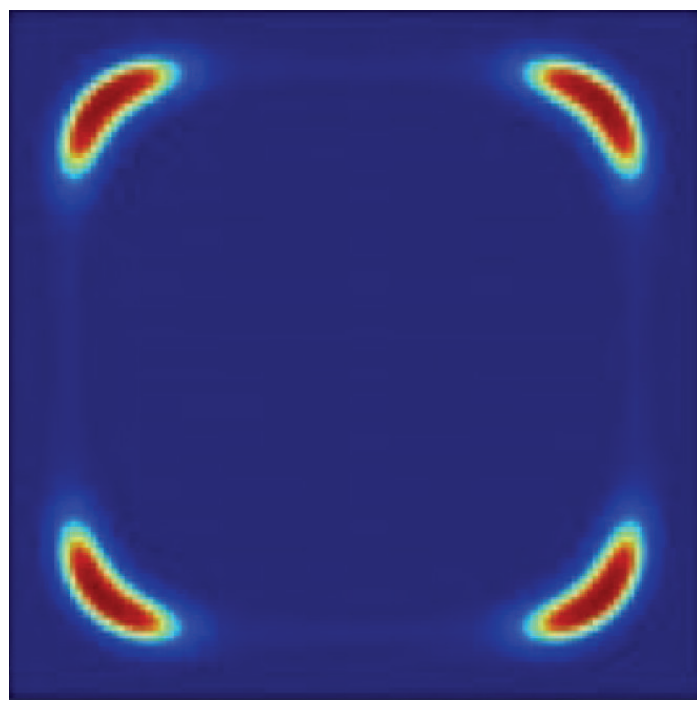

(a)

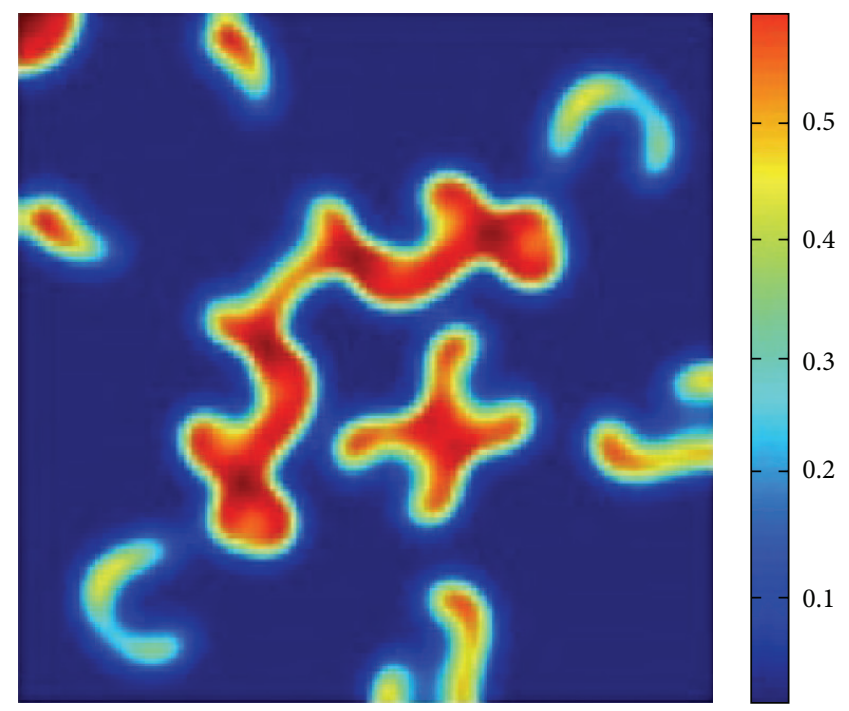

(b)

FIGURE 2: Snapshots of contour pictures of the vegetation at $t=1000$ with noise. Other parameters' values are the same as in Figure 1(a). (a) $\varepsilon=0.001$ and $\tau=5$; (b) $\varepsilon=0.1$ and $\tau=11$.

Figures 4 and 5. One can see that, for $v=0$, small values of noise intensity and temporal correlation cannot induce patch invasion. And for $v \neq 0$, one can choose the appropriate values of noise intensity and temporal correlation to ensure emergence of chaotic patterns.

3.3. Frequency Locking of Systems (2a) and (2b) with Noise and Periodic Force. It is well known that an external periodic force applied to a nonlinear pendulum can cause the pendulum to become entrained at a frequency which is rationally related to the applied frequency, a phenomenon known as frequency locking. It is useful to reveal the complexity of the ecosystem.
It can be found that systems (2a) and (2b) produce oscillations about period $T_{\text {out }}$ with respect to the external period $T_{\text {in }}=2 \pi / \omega$; this phenomenon is called frequency locking or resonant response, that is, when the system produces one spike within each of the $F(F=1,2,3, \ldots)$ periods of the external force, that is, $F: 1$ resonant response. In the present paper, the output period $T_{\text {out }}$ is defined as follows: $T_{i}$ is the time interval between the $i$ th spike and the $i+1$ th spike. $q$ spikes are taken into account and their average value is $T_{\text {out }}$, where $T_{\text {out }}=\sum_{i=1}^{q} T_{i} /(q-1)[33]$.

It is checked by numerical simulations that when systems (1a) and (1b) are only combined with periodic forcing, there 


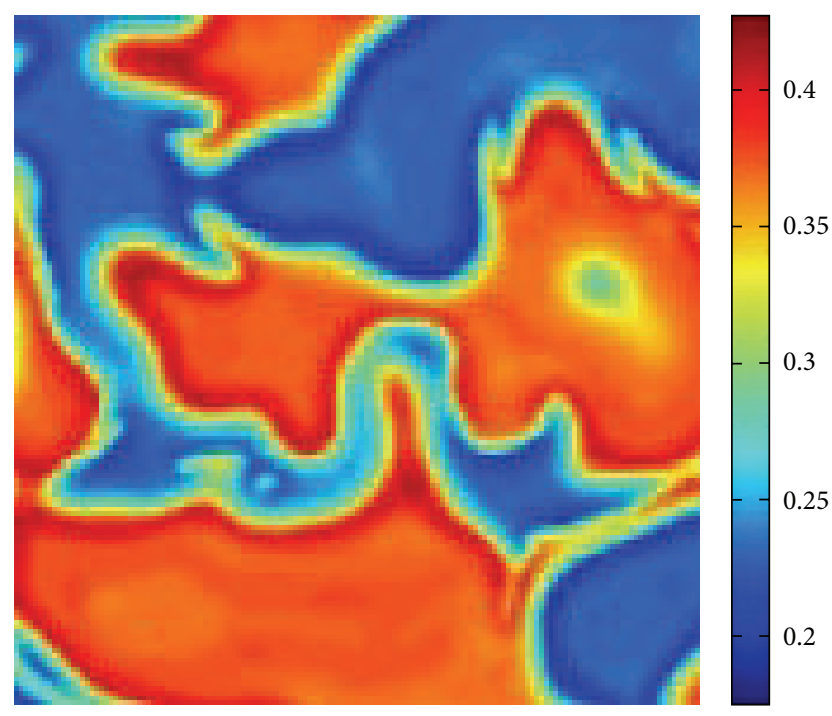

FIGURE 3: Snapshots of contour pictures of the vegetation at $t=1000$ with $\varepsilon=0.07$ and $\tau=6$. Other parameters' values are the same as in Figure 1(b).

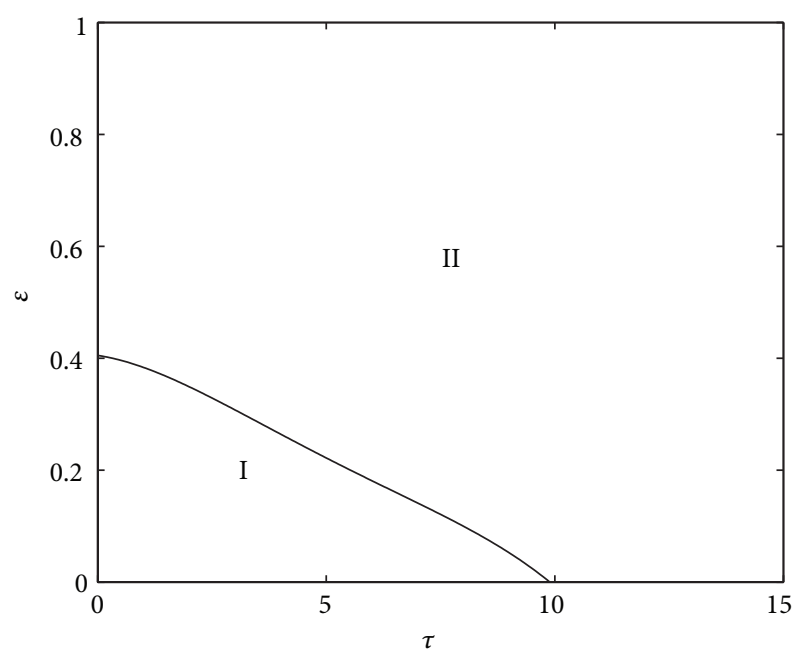

FIGURE 4: Regions of pattern structures of systems (la) and (1b) with respect to noise intensity and temporal correlation for $v=0$. I: no patch invasion; II: patch invasion.

are no frequency locking phenomena. As a result, we do not show the dynamical behavior of systems (1a) and (1b) only with periodic forcing. When there are seasonal rainfall and noise term, we show that there are 1:1 and 2:1 frequency locking in the systems (2a) and (2b), which we plot in Figures 6 and 7. Note that the initial conditions are chosen as in the following form:

$$
\begin{gathered}
n(r, 0)=n^{*}+\phi_{1} x+\phi_{2} y+\varphi, \\
w(r, 0)=w^{*},
\end{gathered}
$$

where $\phi_{1}, \phi_{2}$, and $\varphi$ are positive constants.

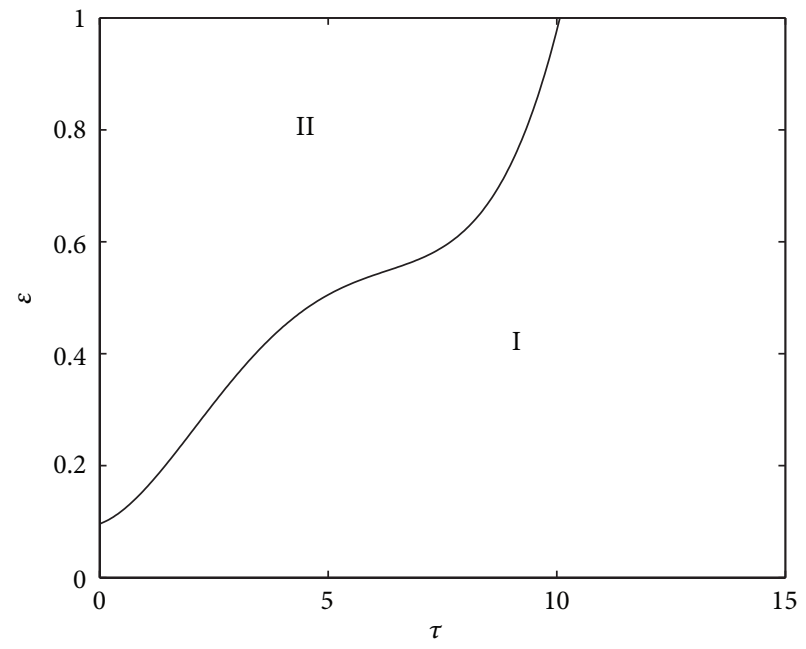

FIGURE 5: Regions of pattern structures of systems (1a) and (1b) with respect to noise intensity and temporal correlation for $v \neq 0$. I: chaotic pattern; II: no chaotic pattern.

Temporal correlation $\tau$ of the colored noise plays important role in the spatial pattern formation and transition of the different patterns. In order to well understand the phase transition by the influence of temporal correlation $\tau$, we give phase diagram of the $\tau-\varepsilon$ parameter space in Figure 8 by performing a series of simulations, that is, fixing $\tau$ and scanning the noise intensity, $\varepsilon$, when the frequency locking evidently changes in the long term. It can be seen from this figure that there are $1: 1$ and $2: 1$ frequency locking in different regions.

\section{Discussion and Conclusion}

In this paper, we investigated a vegetation model combined with seasonal rainfall, noise, and spatial diffusion. By performing a series of numerical simulations, we found that there was emergence of pattern transition from stationary pattern to patch invasion. What is more, chaotic pattern will appear if noise intensity is large. And for the system with both noise and periodic forcing, it exhibits frequency-locking phenomena. The results showed that noise and seasonal rainfall play an important role in vegetation patterns.

Climate fluctuation is also considered as a source of vegetation spatial pattern, which means that all the parameters in systems (2a) and (2b) can show temporal and spatial variations. And it is believed that climate effect can enhance the likelihood of catastrophic shifts to the desert state or control the transitions between preferential states in bistable dynamics [34-36]. Furthermore, in [37], it was shown that static disorder in terms of environmental variability had influence on the pattern dynamics in a spatially ecological system. Then one may want to check whether this phenomenon can occur in vegetation systems. These issues need to be well addressed in the further investigation.

The mechanisms inducing the change of structure or dynamics of vegetation populations are among the most 


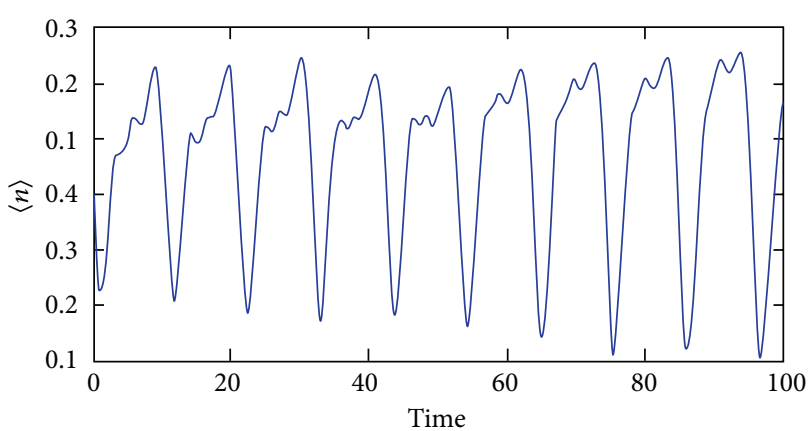

(a)

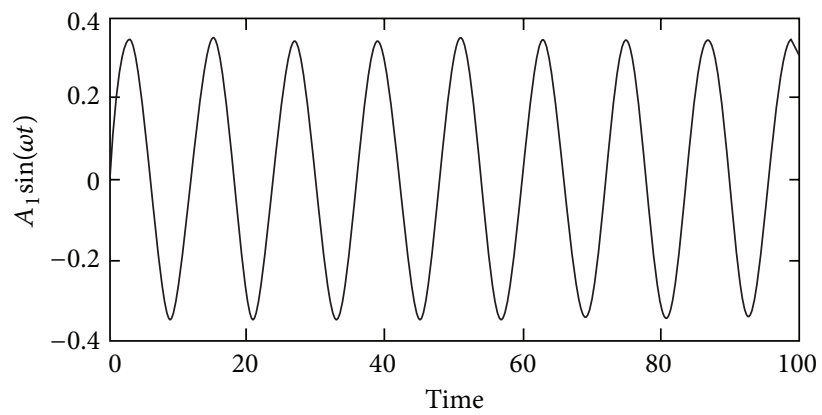

(b)

FIGURE 6: The 1:1 frequency-locking oscillation with the values of the parameters $A_{1}=0.35, \omega=\pi / 6, \varepsilon=0.11$, and $\tau=3$. (a) The mean concentration of vegetation; (b) periodic forces.

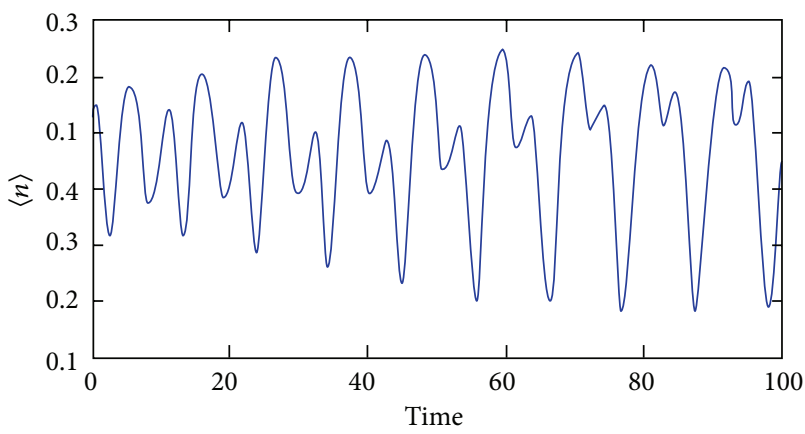

(a)

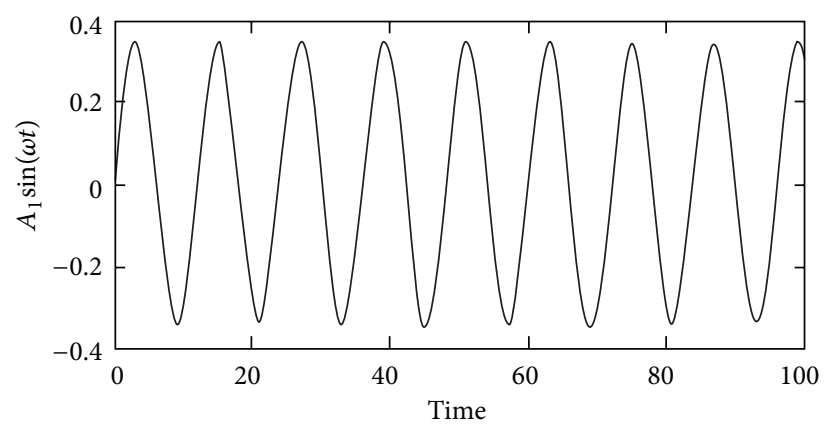

(b)

Figure 7: The $2: 1$ frequency-locking oscillation with the values of the parameters $A_{1}=0.35, \omega=\pi / 6, \varepsilon=0.3$, and $\tau=11$. (a) The mean concentration of vegetation; (b) periodic forces.

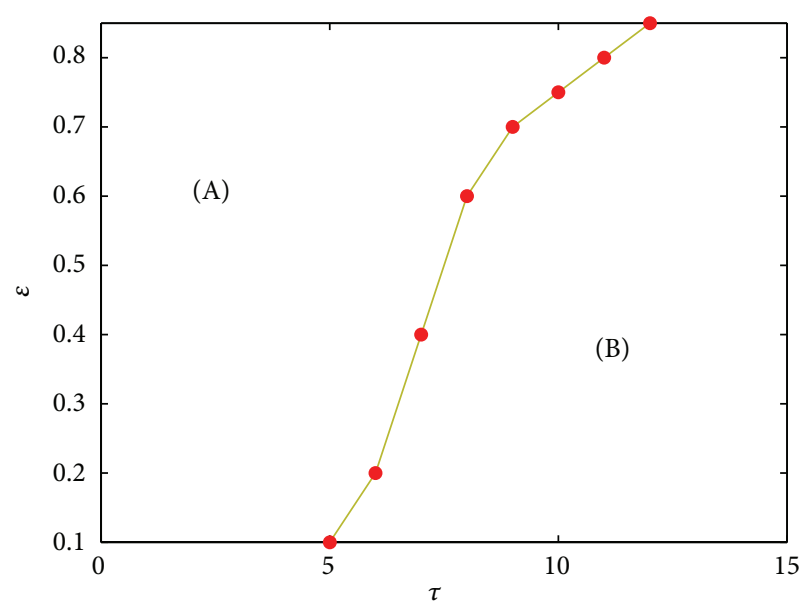

Figure 8: Phase diagram in $\tau-\varepsilon$ parameter space with $A_{1}=0.35$ and $\omega=\pi / 6$. Regions (A) and (B) are corresponding to the $1: 1$ and $2: 1$ frequency locking.

challenging research areas in ecology [38-40]. However, a variety of rich behaviors observed in vegetation populations are far from being well understood [19]. In this sense, rich dynamical behaviors emerging from our work contribute to a better understanding of wetland ecosystems. From an ecological standpoint, the results reported in this paper indicate that noise and external forcing can cause patterns transition in vegetation dynamics which implies that they may induce the onset of desertification. As a result, we need to take measures to decrease stochastic factors and change irrigation to protect vegetation.

\section{Conflict of Interests}

The authors declare that there is no conflict of interests regarding the publication of this paper.

\section{References}

[1] M. Scheffer, A. H. Bakema, and F. G. Wolterboer, "MEGAPLANT: a simulation model of the dynamics of submerged plants," Aquatic Botany, vol. 45, no. 4, pp. 341-356, 1993.

[2] B. T. Grenfell, B. M. Bolker, and A. Kleczkowski, "Seasonality and extinction in chaotic metapopulations," Proceedings of the Royal Society B, vol. 259, no. 1354, pp. 97-103, 1995.

[3] A. A. King and W. M. Schaffer, "The geometry of a population cycle: a mechanistic model of snowshoe hare demography," Ecology, vol. 82, no. 3, pp. 814-830, 2001. 
[4] S. Altizer, A. Dobson, P. Hosseini, P. Hudson, M. Pascual, and P. Rohani, "Seasonality and the dynamics of infectious diseases," Ecology Letters, vol. 9, no. 4, pp. 467-484, 2006.

[5] V. Guttal and C. Jayaprakash, "Self-organization and productivity in semi-arid ecosystems: implications of seasonality in rainfall," Journal of Theoretical Biology, vol. 248, no. 3, pp. 490500, 2007.

[6] A. Provata, I. M. Sokolov, and B. Spagnolo, "Editorial: ecological complex systems," European Physical Journal B, vol. 65, no. 3, pp. 307-314, 2008.

[7] O. Richter, "Spatio-temporal patterns of gene flow and dispersal under temperature increase," Mathematical Biosciences, vol. 218, no. 1, pp. 15-23, 2009.

[8] G. Q. Sun, Z. Jin, L. Li, and Q. X. Liu, "The role of noise in a predator-prey model with Allee effect," Journal of Biological Physics, vol. 35, pp. 185-196, 2009.

[9] G.-Q. Sun, L. Li, Z. Jin, and B.-L. Li, "Effect of noise on the pattern formation in an epidemic model," Numerical Methods for Partial Differential Equations, vol. 26, no. 5, pp. 1168-1179, 2010.

[10] J. G. Vilar and R. V. Sole, "Effects of noise in symmetric twospecies competition," Physical Review Letters, vol. 80, pp. 40994102, 1998.

[11] Q. X. Liu and Z. Jin, "Resonance and frequency-locking phenomena in spatially extended phytoplankton-zooplankton system with additive noise and periodic forces," Journal of Statistical Mechanics, vol. 5, Article ID P05011, 2008.

[12] F. Rao, W. Wang, and Z. Li, "Spatiotemporal complexity of a predator-prey system with the effect of noise and external forcing," Chaos, Solitons \& Fractals, vol. 41, no. 4, pp. 1634-1644, 2009.

[13] G.-Q. Sun, Z. Jin, Q.-X. Liu, and B.-L. Li, "Rich dynamics in a predator-prey model with both noise and periodic force," BioSystems, vol. 100, no. 1, pp. 14-22, 2010.

[14] O. Lejeune, M. Tlidi, and P. Couteron, "Localized vegetation patches: a self-organized response to resource scarcity," Physical Review E, vol. 66, no. 1, Article ID 010901, 2002.

[15] S. B. Boaler and C. A. H. Hodge, "Vegetation stripes in Somaliland," Journal of Ecology, vol. 50, pp. 465-474, 1962.

[16] F. T. Maestre and J. Cortina, "Spatial patterns of surface soil properties and vegetation in a Mediterranean semi-arid steppe," Plant and Soil, vol. 241, no. 2, pp. 279-291, 2002.

[17] C. A. Klausmeier, "Regular and irregular patterns in semiarid vegetation,” Science, vol. 284, no. 5421, pp. 1826-1828, 1999.

[18] M. Rietkerk, S. C. Dekker, P. C. De Ruiter, and J. Van De Koppel, "Self-organized patchiness and catastrophic shifts in ecosystems," Science, vol. 305, no. 5692, pp. 1926-1929, 2004.

[19] M. Rietkerk and J. van de Koppel, "Regular pattern formation in real ecosystems," Trends in Ecology and Evolution, vol. 23, no. 3, pp. 169-175, 2008.

[20] C. Valentin, J. M. D’Herbès, and J. Poesen, “Soil and water components of banded vegetation patterns," Catena, vol. 37, no. 1-2, pp. 1-24, 1999.

[21] J. Von Hardenberg, E. Meron, M. Shachak, and Y. Zarmi, "Diversity of vegetation patterns and desertification," Physical Review Letters, vol. 87, no. 19, Article ID 198101, 2001.
[22] K. L. Kirk and J. J. Gilbert, "Variation in herbivore response to chemical defenses: zooplankton foraging on toxic cyanobacteria," Ecology, vol. 73, no. 6, pp. 2208-2217, 1992.

[23] T. Reichenbach, M. Mobilia, and E. Frey, "Noise and correlations in a spatial population model with cyclic competition," Physical Review Letters, vol. 99, no. 23, Article ID 238105, 2007.

[24] T. Reichenbach, M. Mobilia, and E. Frey, "Mobility promotes and jeopardizes biodiversity in rock-paper-scissors games," Nature, vol. 448, no. 7157, pp. 1046-1049, 2007.

[25] R. Mankin, A. Ainsaar, A. Haljas, and E. Reiter, "Trichotomousnoise-induced catastrophic shifts in symbiotic ecosystems," Physical Review E, vol. 65, no. 5, Article ID 051108, 2002.

[26] R. Mankin, A. Sauga, A. Ainsaar, A. Haljas, and K. Paunel, "Colored-noise-induced discontinuous transitions in symbiotic ecosystems," Physical Review E, vol. 69, no. 6, Article ID 061106, 2004.

[27] D. J. Higham, "An algorithmic introduction to numerical simulation of stochastic differential equations," SIAM Review, vol. 43 , no. 3, pp. 525-546, 2001.

[28] G. Q. Sun, Z. Jin, Q. X. Liu, and L. Li, "Dynamical complexity of a spatial predator-prey model with migration," Ecological Modelling, vol. 219, pp. 248-255, 2008.

[29] G. Q. Sun, L. Li, Z. Jin, and B. L. Li, "Pattern formation in a spatial plant-wrack model with tide effect on the wrack," Journal of Biological Physics, vol. 36, pp. 161-174, 2010.

[30] G.-Q. Sun, J. Li, B. Yu, and Z. Jin, "Noise induced pattern transition in a vegetation model," Applied Mathematics and Computation, vol. 221, pp. 463-468, 2013.

[31] C. Zimmer, "Life after chaos," Science, vol. 284, pp. 83-86, 1999.

[32] B. Blasius, A. Huppert, and L. Stone, "Complex dynamics and phase synchronization in spatially extended ecological systems," Nature, vol. 399, no. 6734, pp. 354-359, 1999.

[33] F. N. Si, Q. X. Liu, J. Z. Zhang, and L. Q. Zhou, "Propagation of travelling waves in sub-excitable systems driven by noise and periodic forcing," The European Physical Journal B, vol. 60, pp. 507-513, 2007.

[34] P. D’Odorico, F. Laio, and L. Ridolfi, "Noise-induced stability in dryland plant ecosystems," Proceedings of the National Academy of Sciences of the United States of America, vol. 102, pp. 10819$10822,2005$.

[35] P. D’Odorico, A. Porporato, and L. Ridolfi, "Transition between stable states in the dynamics of soil development," Geophysical Research Letters, vol. 28, pp. 595-598, 2001.

[36] V. Isham, D. R. Cox, I. Rodríguez-Iturbe, A. Porporato, and S. Manfreda, "Representation of space-time variability of soil moisture," Proceedings of the Royal Society A, vol. 461, no. 2064, pp. 4035-4055, 2005.

[37] M. Fras and M. Gosak, "Spatiotemporal patterns provoked by environmental variability in a predator-prey model," Biosystems, vol. 114, pp. 172-177, 2013.

[38] L. Ridolfi, P. D’Odorico, and F. Laio, "Vegetation dynamics induced by phreatophyte-aquifer interactions," Journal of Theoretical Biology, vol. 248, no. 2, pp. 301-310, 2007.

[39] D. Tilman, Dynamics and Structure of Plant Communities, Princeton University Press, Princeton, NJ, USA, 1988.

[40] J. P. Grover, Resource Competition, Chapman and Hall, New York, NY, USA, 1997. 


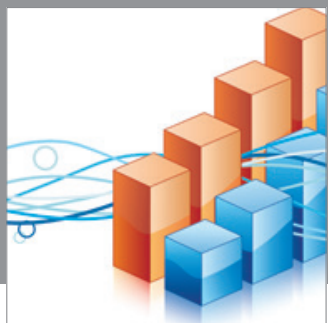

Advances in

Operations Research

mansans

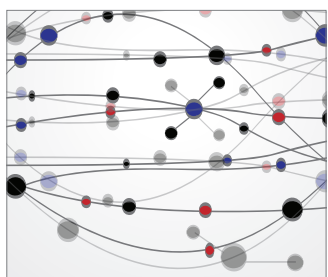

The Scientific World Journal
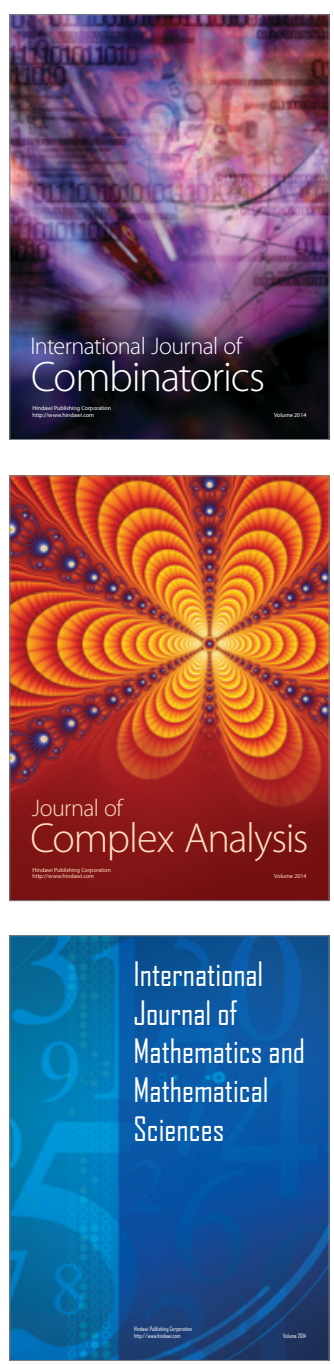
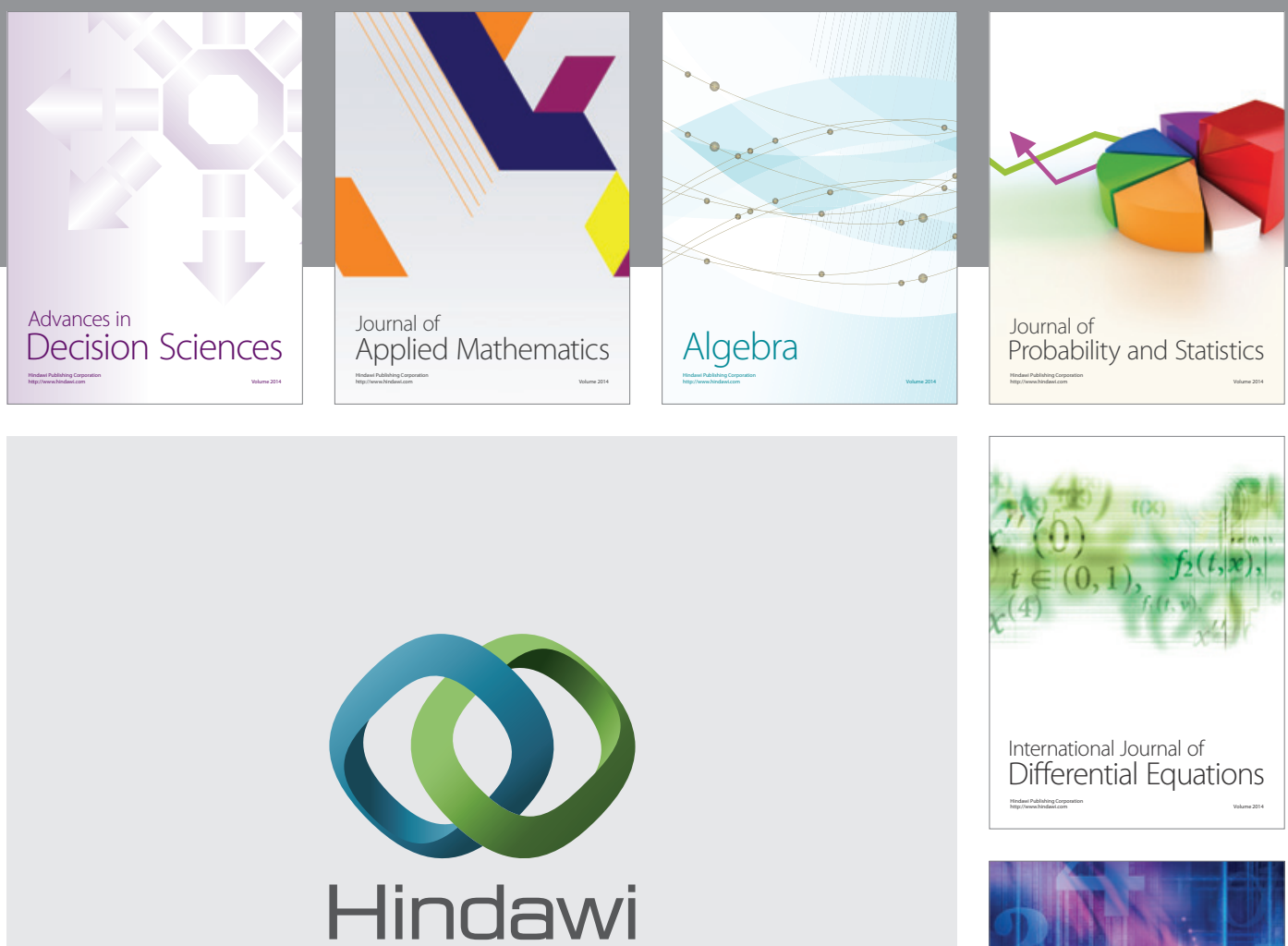

Submit your manuscripts at http://www.hindawi.com
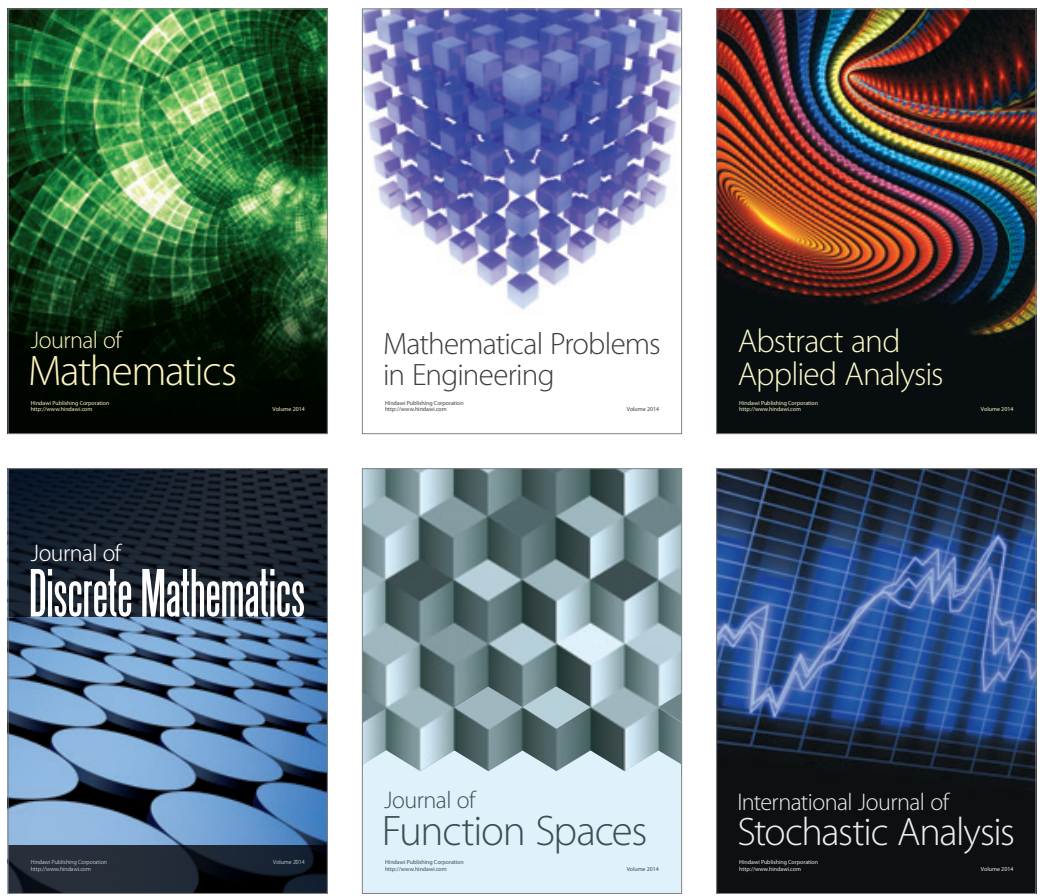

Journal of

Function Spaces

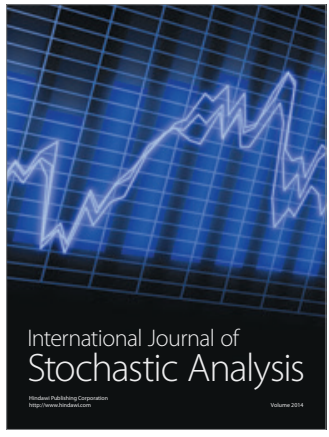

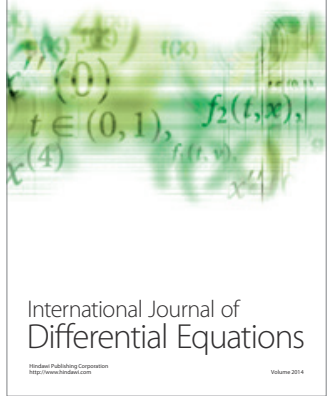
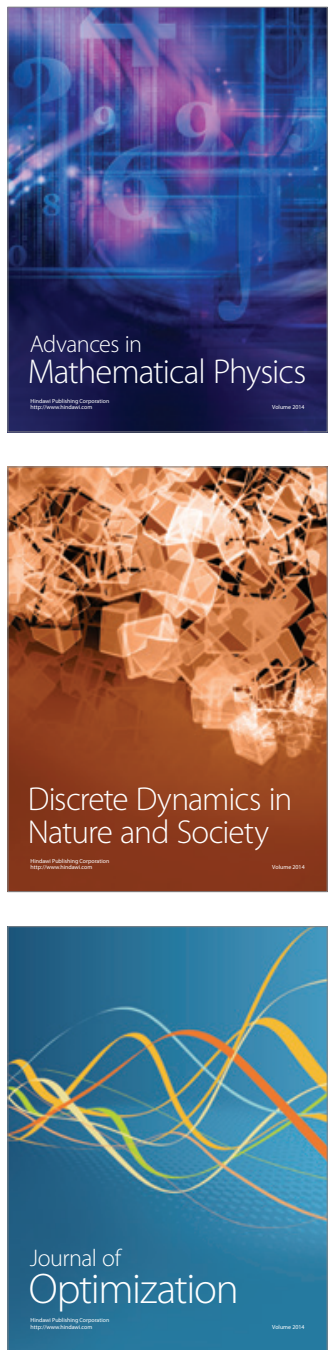